\title{
SPECTRAL ASYMPTOTICS FOR SPINOR LAPLACIANS AND MULTIPLICITIES
}

\author{
HENRI MOSCOVICI
}

\begin{abstract}
We use Getzler's formula for the heat kernel of the spinor Laplacian to derive information about the asymptotic distribution of multiplicities in the quasi-regular representation of a semisimple Lie group $G$ modulo a cocompact discrete subgroup $\Gamma$.
\end{abstract}

0. Introduction. Let $G$ be a connected semisimple Lie group with finite center and let $\Gamma$ be a discrete subgroup of $G$ such that $\Gamma \backslash G$ is compact. It is well known that the right regular representation $R_{\Gamma}$ of $G$ on $L^{2}(\Gamma \backslash G)$ splits into a countable direct sum of irreducible unitary representations and that for each class $\pi \in \hat{G}$ occurring in this decomposition the multiplicity $N_{\Gamma}(\pi)$ is finite. The integers $N_{\Gamma}(\pi)$ have been, and still are, the subject of a great deal of investigation. One direction consists in studying their distribution as $\pi$ "approaches infinity" in $\hat{G}$. This is achieved by relating the $N_{\Gamma}(\pi)$ to the traces of suitable heat operators on $M=$ $\Gamma \backslash G / K$ and then studying their asymptotic expansions. Here $K$ is a maximal compact subgroup of $G$. Also, we shall assume for simplicity that $\Gamma$ is torsion free and therefore $M$ is a smooth compact manifold.

Thus, using the connection Laplacian on $M$ naturally associated to a finitedimensional representation $\tau$ of $K$, Wallach proves in [7] that $(0.1)$

$$
\sum_{\pi \in \hat{G}} N_{\Gamma}(\pi)\left\langle\pi_{K}, \tau\right\rangle e^{t \chi_{\pi}(\Omega)}=(4 \pi t)^{-m / 2} \operatorname{dim}(\tau) \operatorname{vol}(M)+o\left(t^{-m / 2}\right) \quad \text { as } t \rightarrow 0^{+},
$$

where $\left\langle\pi_{K}, \tau\right\rangle$ is the intertwining number of $\pi$ restricted to $K$ and $\tau, \chi_{\pi}$ is the infinitesimal character of $\pi, \Omega$ stands for the Casimir element of $G, m=\operatorname{dim}(M)$ and $\operatorname{vol}(M)$ is the volume of $M$ (with respect to a natural Riemannian structure).

In fact, one has an asymptotic expansion of the form

$$
\sum_{\pi \in \hat{G}} N_{\Gamma}(\pi)\left\langle\pi_{K}, \tau\right\rangle e^{t \chi_{\pi}(\Omega)} \sim \sum_{k=0}^{\infty} a_{k}(\tau) t^{k-m / 2} \text { as } t \rightarrow 0^{+}
$$

but, except when $\operatorname{rank}(G / K)=1$ (see [4]), not much is known about the coefficients $a_{k}(\tau), k>0$.

In this note we show that for $0 \leq k \leq m / 2$ the coefficients $a_{k}$, viewed as functions of $\tau$, satisfy a series of equations which can be regarded as asymptotic analogues of the alternating sum formulas for multiplicities in [5]. This information is extracted from a limit formula of Getzler for the heat kernel of the Dirac operator (cf. [2, 3]).

Received by the editors August 19, 1985 and, in revised form, February 4, 1986.

1980 Mathematics Subject Classification (1985 Revision). Primary 22E46, 58G25.

Research supported in part by the National Science Foundation. 
1. A consequence of Getzler's formula. Given an inner product space $V$ over $\mathbf{R}$ we denote by $C(V)$ the associated Clifford algebra, defined as in [2], and by $\sigma: C(V) \rightarrow \bigwedge V, \theta: \bigwedge V \rightarrow C(V)$ the canonical isomorphisms (of vector spaces) betwen the Clifford algebra and the exterior algebra of $V$, with $\theta=\sigma^{-1}$. According to whether $m=\operatorname{dim} M$ is even or odd, $\mathbf{C}(V)=C(V) \otimes_{\mathbf{R}} \mathbf{C}$, resp $\mathbf{C}^{\text {ev }}(V)=$ $\theta\left(\bigwedge_{\mathbf{C}}^{\mathrm{ev}} V\right)$, is a simple algebra over $\mathbf{C}$ and thus has a unique simple module $S(V)$ of dimension $2^{m / 2}$, resp. $2^{[(m+1) / 2]}$, called the space of spinors over $V$. In what follows we shall identify $\mathbf{C}(V)$, resp. $\mathbf{C}^{\text {ev }}(V)$, with End $S(V)$. We recall that $S(V)$ can be equipped with an inner product such that each $v \in V$ acts as a selfadjoint operator on $S(V)$. We shall need the following elementary result, whose proof is similar to that of Theorem 1.8 in [2] and will be omitted.

LEMma. Let $\omega \in \bigwedge_{\mathbf{C}}^{2 l} V$ and $h \in \operatorname{End} S(V)$. Then

$$
\operatorname{Tr}(\theta(\omega) h)=(-1)^{l} \operatorname{dim} S(V)\langle\sigma(h), \bar{\omega}\rangle .
$$

We now consider a compact spin manifold $M$, of dimension $m$, and denote by $S(T M)$ its spin bundle. End $S(T M)$ will be identified with $\mathbf{C}(T M)$ if $m$ is even, respectively with $\mathbf{C}^{\text {ev }}(T M)$ when $m$ is odd. We shall also identify $T M$ and $T^{*} M$ via the Riemannian metric. The above isomorphisms $\sigma$ and $\theta$ will then induce bundle isomorphisms $\sigma: \mathbf{C}(T M) \rightarrow \bigwedge_{\mathbf{C}} T^{*} M, \theta: \bigwedge_{\mathbf{C}} T^{*} M \rightarrow \mathbf{C}(T M)$.

Let $E$ be a Hermitian vector bundle $M$, with connection $\nabla^{E}$. The Dirac operator $D_{E}$, acting on the space of $C^{\infty}$ sections $\Gamma(S(T M) \otimes E)$, is defined in terms of a local orthonormal frame $\left\{e_{1}, \ldots, e_{m}\right\}$ by the expression

$$
D_{E}=\sum_{j=1}^{m} \theta\left(e_{j}\right) \otimes I\left(\nabla_{e_{j}}^{S} \otimes I+I \otimes \nabla_{e_{j}}^{E}\right)
$$

where $\nabla^{S}$ is the connection on $S(T M)$ induced by the Riemannian connection on $T M$. With the present definition of the Clifford algebra, $D_{E}$ is skew-adjoint and therefore the associated heat semigroup is $e^{t D_{E}^{2}}$.

Let $K_{t}(x, y)$ be the Schwartz kernel of $e^{t D_{E}^{2}}$. For each $x \in M, K_{t}(x, x)$ is an endomorphism of $S\left(T_{x} M\right) \otimes E_{x}$ and so $\operatorname{Tr}_{E_{x}} K_{t}(x, x) \in \operatorname{End} S\left(T_{x} M\right)$. We now define a (nonhomogeneous) form $k_{t}(x)=\sum_{j=0}^{m} k_{t}^{(j)}(x)$ on $M$ by

$$
k_{t}(x)=\sigma\left(\operatorname{Tr}_{E_{x}} K_{t}(x, x)\right) .
$$

Getzler's main result in $[\mathbf{3}]$ implies that

$$
\lim _{\varepsilon \rightarrow 0^{+}} \sum_{j=0}^{m} \varepsilon^{m-j} k_{\varepsilon^{2} t}^{(j)}(x)=(4 \pi t)^{-m / 2} \operatorname{ch}\left(t R_{E}\right)(x) \wedge \hat{A}(t R)(x),
$$

where $R \in \Gamma\left(\bigwedge^{2} T_{\mathbf{C}}^{*} M \otimes\right.$ End $\left.T M\right)$ is the Riemannian curvature of $M, R_{E} \in$ $\Gamma\left(\bigwedge^{2} T_{\mathbf{C}}^{*} M \otimes\right.$ End $\left.E\right)$ is the curvature of the connection $\nabla^{E}$,

$$
\hat{A}(R)=\operatorname{det}\left(\frac{R / 2}{\sinh (R / 2)}\right)^{1 / 2} \in \Gamma\left(\bigwedge^{\mathrm{ev}} T_{\mathbf{C}}^{*} M\right)
$$

and

$$
\operatorname{ch}\left(R_{E}\right)=\operatorname{Tr}\left(e^{R_{E}}\right) \in \Gamma\left(\bigwedge^{\mathrm{ev}} T_{\mathbf{C}}^{*} M\right)
$$


Taking in both sides of (1.1) the scalar product with $\bar{\omega}(x)$, where $\omega$ is a $2 l$-form on $M$, one obtains

$$
\lim _{t \rightarrow 0^{+}} \varepsilon^{m-2 l}\left\langle k_{\varepsilon^{2} t}(x), \bar{\omega}(x)\right\rangle=(4 \pi t)^{-m / 2} t^{l}\left\langle\operatorname{ch}\left(R_{E}\right)(x) \wedge \hat{A}(R)(x), \bar{\omega}(x)\right\rangle .
$$

Now, due to the above lemma, one can rewrite this as

$$
\lim _{t \rightarrow 0^{+}} t^{m / 2-l} \operatorname{Tr}\left((\theta(\omega)(x) I) K_{t}(x, x)=(-1)^{l} C_{m}\left\langle\operatorname{ch}\left(R_{E}\right)(x) \wedge \hat{A}(R)(x), \bar{\omega}(x)\right\rangle,\right.
$$

where

$$
C_{m}=2^{-[(m+1) / 2]} \pi^{-m / 2} .
$$

Since $x \longmapsto(\theta(\omega)(x) \otimes I) K_{t}(x, x) \in$ End $S\left(T_{x} M\right) \otimes E_{x}$ is the diagonal restriction of the Schwartz kernel of the operator $(\theta(\omega) \otimes I) e^{t D_{E}^{2}}$, by integrating (1.2) over $M$ one obtains the following statement.

Proposition. For any $2 l$-form $\omega$ on $M$, one has.

$$
\lim _{t \rightarrow 0^{+}} t^{m / 2-l} \operatorname{Tr}((\theta(\omega) \otimes I)) e^{t D_{E}^{2}}=(-1)^{l} C_{m} \int_{M} \operatorname{ch}\left(R_{E}\right) \wedge \hat{A}(R) \wedge * \omega .
$$

Let us note that for the 0 -form $\omega=1$ this is just Weyl's formula for spinor Laplacians, while for $l=m / 2\left(m\right.$ even) and $i^{-l} \omega=$ the volume form, one gets the index formula for Dirac operators.

2. Application to multiplicities. We shall now specialize the above result to the case of a compact locally symmetric space $M=\Gamma \backslash G / K$, where $G, K$ and $\Gamma$ are as in the introduction. Let $\mathfrak{g}$ (resp. $\mathfrak{k}$ ) be the Lie algebra of $G$ (resp. $K$ ) and let $\mathfrak{p}$ be the orthogonal of $\mathfrak{k}$ with respect to the Cartan-Killing form $B$. We endow $M$ with the Riemannian metric obtained from $B \mid \mathfrak{p} \times \mathfrak{p}$ via the identification of $\mathfrak{p}$ with the tangent space at $o=1 \cdot K$ to $G / K$.

Let $\mathfrak{t}$ be a maximal abelian subalgebra of $\mathfrak{k}$ and let $\mathfrak{h}$ be the centrlizer of $\mathfrak{t}$ in $\mathfrak{g}$. Then $\mathfrak{h}$ is a Cartan subalgebra of $\mathfrak{g}$. Let $\Phi$ be the root system of $\left(\mathfrak{g}_{\mathbf{C}}, \mathfrak{h}_{\mathbf{C}}\right)$ and let $\Phi_{k}$ be the root system of $\left(\mathfrak{k}_{\mathbf{C}}, \mathrm{t}_{\mathbf{C}}\right)$. We fix, once and for all, a set of positive roots $\Psi_{k}$ for $\Phi_{k}$ and a set of positive roots $\Psi$ for $\Phi$, in a compatible fashion. As usual, we denote by $\rho, \rho_{k}$, and $\rho_{n}$ the half-sum of the roots in $\Psi, \Psi_{k}$, and $\Psi_{n}=\Psi-\Psi_{k}$ respectively. Let $T$ be the maximal torus of $K$ with Lie algebra $t$. The dual group $\hat{T}$ will be identified, via exponentiation, to a lattice $L_{T} \subset i t^{*}$.

The spin module associated to $(\mathfrak{p},\langle\rangle$,$) will be denoted S$. It is, in particular, a $\mathfrak{k}$-module. We do not postulate the existence of a $G$-invariant spin structure on the symetric space $G / K$, and thus $S$ need not be a $K$-module. Consider, however, an irreducible $\mathfrak{k}$-module $V_{\nu}$ whose highest weight $\nu \in i \mathfrak{t}^{*}$ satisfies the condition

$$
\nu+\rho_{n} \in L_{T} .
$$

Since every weight of $S$ differs from $\rho_{n}$ by a sum of roots, (2.1) is easily seen to guarantee the fact that the representation of $\mathfrak{k}$ on $S \otimes V_{\nu}$ lifts to a representation of $K$. This representation of $K$, in turn, gives rise first to a homogeneous bundle over $G / K$ and then, by passing to $\Gamma$-orbits, to a vector bundle over $M$ which will be denoted $S(M, \nu)$. This bundle comes equipped with a Hermitian structure, induced by the $K$-invariant inner product on $S \otimes V_{\nu}$ and with a unitary connection $\nabla^{(S, \nu)}$, inherited from that of the principal bundle $G \rightarrow G / K$ (defined by the splitting 
$\mathfrak{g}=\mathfrak{k} \oplus \mathfrak{p})$. We can therefore form the twisted Dirac operator $D_{\nu}: \Gamma(S(M, \nu)) \rightarrow$ $\Gamma(S(M, \nu))$. Explicitly, after identifying $\Gamma(S(M, \nu))$ with the subspace $\left(C^{\infty}(\Gamma / G) \otimes\right.$ $\left.S \otimes V_{\nu}\right)^{K}$ of all $K$-invariant elements in $C^{\infty}(\Gamma / G) \otimes S \otimes V_{\nu}, D_{\nu}$ is given by the formula

$$
D_{\nu}=\sum_{j=1}^{m} R_{\Gamma}\left(X_{j}\right) \otimes \theta\left(X_{j}\right) \otimes I,
$$

where $\left\{X_{1}, \ldots, X_{m}\right\}$ is an orthonormal basis for $\mathfrak{p}$. Moreover (see $[6], \S 3$ ),

$$
-D_{\nu}^{2}=-R_{\Gamma}(\Omega) \otimes I \otimes I+\left(\left\|\nu+\rho_{k}\right\|^{2}-\|\rho\|^{2}\right) I \otimes I \otimes I,
$$

where $\Omega$ is the Casimir element of $\mathfrak{g}$.

We recall that $\Lambda \mathfrak{p}_{\mathrm{C}}^{*}$ (resp. $\Lambda^{\mathrm{ev}} \mathfrak{p}_{\mathrm{C}}^{*}$, if $m$ is odd) and $\operatorname{End}(S)$ are isomorphic as $\mathrm{SO}(\mathfrak{p})$-modules. In particular $\omega \in \wedge^{\mathrm{ev}} \mathfrak{p}_{\mathrm{C}}^{*}$ is $K$-invariant if and only if $\theta(\omega) \in$ $\operatorname{End}_{K}(S)$.

Consider now a unitary representation $\pi$ of $G$ on a Hilbert space $\mathfrak{H}_{\pi}$. Then

$$
I \otimes \theta(\omega) \otimes I: \mathfrak{H}_{\pi} \otimes S \otimes V_{\nu} \rightarrow \mathfrak{H}_{\pi} \otimes S \otimes V_{\nu}
$$

commutes with the (tensor product) action of $K$, and therefore restricts to an operator

$$
\theta_{\pi, \nu}(\omega):\left(\mathfrak{H}_{\pi} \otimes S \otimes V_{\nu}\right)^{K} \rightarrow\left(\mathfrak{H}_{\pi} \otimes S \otimes V_{\nu}\right)^{K},
$$

where the superscript $K$ signifies passage to $K$-invariant elements. Note that if $\pi$ is irreducible, $\left(\mathfrak{H}_{\pi} \otimes S \otimes V_{\nu}\right)^{K}$ has finite dimension.

Let $r \in \Lambda^{2} \mathfrak{p}_{\mathbf{C}}^{*} \otimes \operatorname{End}(\mathfrak{p})$ and $r_{\nu} \in \Lambda^{2} \mathfrak{p}_{\mathbf{C}}^{*} \otimes \operatorname{End}\left(V_{\nu}\right)$ be defined as follows

$$
\begin{aligned}
& r=-\sum_{1 \leq i, j \leq m} \operatorname{ad}\left[X_{i}, X_{j}\right] \otimes \xi_{i} \wedge \xi_{j}, \\
& r_{\nu}=-\sum_{1 \leq i, j \leq m} \tau_{\nu}\left[X_{i}, X_{j}\right] \otimes \xi_{i} \wedge \xi_{j},
\end{aligned}
$$

where $\left\{X_{1}, \ldots, X_{m}\right\}$ is an orthonormal basis of $\mathfrak{p},\left\{\xi_{1}, \ldots, \xi_{m}\right\}$ is it dual basis for $\mathfrak{p}^{*}$, and $\tau_{\nu}$ denotes the representation of $K$ on $V_{\nu}$. It is easy to check that $r$ and $r_{\nu}$ are independent of the orthonormal basis, and also that they are $K$-invariant. We then form

$$
\hat{A}(r)=\operatorname{det}\left(\frac{r / 2}{\sinh (r / 2)}\right)^{1 / 2} \in\left(\bigwedge^{\mathrm{ev}} \mathfrak{p}_{\mathrm{C}}^{*}\right)^{K}
$$

and

$$
\operatorname{ch}\left(r_{\nu}\right)=\operatorname{Tr}_{V_{\nu}}\left(e^{r_{\nu}}\right) \in\left(\bigwedge^{\mathrm{ev}} \mathfrak{p}_{\mathrm{C}}^{*}\right)^{K} .
$$

Finally, we denote

$$
\hat{G}_{\Gamma, \nu}=\left\{\pi \in \hat{G} ; N_{\Gamma}(\pi)>0, \operatorname{dim}\left(\mathfrak{H}_{\pi} \otimes S \otimes V_{\nu}\right)^{K}>0\right\}
$$

and recall that $\chi_{\pi}$ stands for the infinitesimal character of $\pi \in \hat{G}$.

THEOREM. For each $\omega \in\left(\bigwedge^{2 l} \mathfrak{p}_{\mathrm{C}}^{*}\right)^{K}$, one has

$$
\begin{aligned}
& \lim _{t \rightarrow 0^{+}} t^{m / 2-l} \sum_{\pi \in \hat{G}_{\Gamma, \nu}} N_{\Gamma}(\pi) \operatorname{Tr} \theta_{\pi, \nu}(\omega) e^{\chi \pi(\Omega) t} \\
&=(-1)^{l} C_{m}\left\langle\operatorname{ch}\left(r_{\nu}\right) \wedge \hat{A}(r), \bar{\omega}\right\rangle \operatorname{vol}(\Gamma \backslash G / K) .
\end{aligned}
$$


ProOF. Let us assume for the moment that the $\mathfrak{k}$-action on $S$ does lift to $K$ and therefore $M$ is a spin manifold. $S(T M)$ is then the bundle induced by $S$. We denote by $E_{\nu}$ the bundle on $M$ induced by $V_{\nu}$ and by $\tilde{\omega}$ the form on $M$ whose lift to $G / K$ is the $G$-invariant form determined by $\omega$. Clearly, $\theta(\tilde{\omega}) \in \operatorname{End} S(T M)$ is just the indomorphism induced by $\theta(\omega) \in \operatorname{End}_{K}(S)$. Furthermore, under the identification of $\Gamma\left(S(T M) \otimes E_{\nu}\right)$ with $\left(C^{\infty}(\Gamma \backslash G) \otimes S \otimes V_{\nu}\right)^{K}$, the mulitplication operator $\theta(\tilde{\omega}) \otimes I$ becomes

$$
\theta_{R_{\Gamma}, \nu}(\omega)=I \otimes \theta(\omega) \otimes I \mid\left(L^{2}(\Gamma \backslash G) \otimes S \otimes V_{\nu}\right)^{K}
$$

Let us also note that, since the Riemannian connection on $T M$ coincides with that induced by the canonical $G$-invariant connection on $T(G / K)$, the Dirac operator $D_{E_{\nu}}$ is precisely the operator $D_{\nu}$ given by (2.2).

From the decomposition of $R_{\Gamma}$ into irreducible components,

$$
L^{2}(\Gamma \backslash G)=\sum_{\pi \in \hat{G}}^{\oplus} N_{\Gamma}(\pi) \mathfrak{H}_{\pi}
$$

it follows that

$$
\left(L^{2}(\Gamma \backslash G) \otimes S \otimes V_{\nu}\right)^{K} \cong \sum_{\pi \in \hat{G}_{\Gamma, \nu}}^{\oplus} N_{\Gamma}(\pi)\left(\mathfrak{H}_{\pi} \otimes S \otimes V_{\nu}\right)^{K} .
$$

So, each $\pi \in \hat{G}_{\Gamma, \nu}$ contributes $N_{\Gamma}(\pi)$ summands of the form $\left(\mathfrak{H}_{\pi} \otimes S \otimes V_{\nu}\right)^{K}$; in turn, each of these summands is invariant under both $D_{\nu}^{2}$ dand $\theta_{R_{\Gamma}, \nu}(\omega)$. Moreover, one has:

$$
D_{\nu}^{2} \mid\left(\mathfrak{H}_{\pi} \otimes S \otimes V_{\nu}\right)^{K}=\left(\chi_{\tau}(\Omega)-\left(\left\|\nu+\rho_{k}\right\|^{2}-\|\rho\|^{2}\right)\right) I \quad(\mathrm{cf.}(2.3))
$$

and

$$
\theta_{R_{\Gamma}, \nu}(\omega) \mid\left(\mathfrak{H}_{\pi} \otimes S \otimes V_{\nu}\right)^{K}=\theta_{\pi, \nu}(\omega)
$$

Therefore,

$$
\operatorname{Tr}\left(\theta_{R_{\Gamma}, \nu}(\omega) e^{t D^{2}}\right)=e^{\left(\|\rho\|^{2}-\left\|\nu+\rho_{k}\right\|^{2}\right) t} \sum_{\pi \in \hat{G}_{\Gamma, \nu}} N_{\Gamma}(\pi) \operatorname{Tr} \theta_{\pi, \nu}(\omega) e^{\chi_{\pi}(\Omega) t},
$$

and thus (2.4) follows immediately from (1.3).

Finally, let us drop the assumption that $S$ integrates to a representation of $K$. The role of the bundle $S(T M) \otimes E$ is then assumed by $S(M, \nu)$, the bundle induced by the $K$-module $S \otimes V_{\nu}$. Now $\operatorname{End} S(M, \nu)$ is induced by $\operatorname{End}\left(S \otimes V_{\nu}\right)=$ $\operatorname{End}(S) \otimes \operatorname{End}\left(V_{\nu}\right)$ and, unlike $S$ and $V_{\nu}$ which are only $k$-modules, both $\operatorname{End}(S)$ and $\operatorname{End}\left(V_{\nu}\right)$ are in fact $K$-modules. With this observation it is easy to see that the arguments in $\S 1$ still apply, giving (1.3) and therefore (2.4).

When $\omega=1,(2.4)$ gives no new information beyond that coming from (0.1). At the opposite extreme, when $l=m / 2$ (assuming $m$ is even) and $i^{-l} \omega=$ the invariant volume form $v$ on $G / K$, the left-hand side of (2.4) is independent of $t$ and coincides with the index of $D_{\nu}$. Thus (see also [1, §1]), (2.4) becomes

$$
\begin{gathered}
\sum_{\pi \in \hat{G}_{\Gamma, \nu}} N_{\Gamma}(\pi)\left(\operatorname{dim}\left(\mathfrak{H}_{\pi} \otimes S^{+} \otimes V_{\nu}\right)^{K}-\operatorname{dim}\left(\mathfrak{H}_{\pi} \otimes S^{-} \otimes V_{\nu}\right)^{K}\right) \\
=C_{m} i^{m / 2}\left\langle\left(\operatorname{ch}\left(r_{\nu}\right) \wedge \hat{A}(r)\right)^{(m)}, v\right\rangle \operatorname{vol}(\Gamma \backslash G / K),
\end{gathered}
$$


which is essentially Miatello's alternating sum formula for multiplicities, associated to the homogeneous symbol of $D_{\nu}$ (cf. [4]). In general, (2.4) can be viewed as interpolating between the two extremes.

Let us finally interpret the theorem in terms of the coefficient functions of the asymptotic expansion (0.2). Let

$$
S=\sum_{i=1}^{N} S_{i}, \quad \sigma=\sum_{i=1}^{N} \sigma_{i}
$$

be the decomposition of $S$, as a k-module, into irreducible components, and let $P_{i}$ denote the orthogonal projection in $\operatorname{End}_{K}(S)$ associated to $S_{i}, 1 \leq i \leq N$. Then

$$
\theta(\omega)=\sum_{i=1}^{N} c^{i}(\omega) P_{i}
$$

with

$$
c^{i}(\omega)=\operatorname{Tr}\left(\theta(\omega) P_{i}\right) / \operatorname{dim} S_{i}
$$

Hence

and therefore

$$
\theta_{\pi, \nu}(\omega)=\sum_{i=1}^{N} c^{i}(\omega) I \otimes P_{i} \otimes I \mid\left(\mathfrak{H}_{\pi} \otimes S \otimes V_{\nu}\right)^{K}
$$

$$
\operatorname{Tr} \theta_{\pi, \nu}(\omega)=\sum_{i=1}^{N} c^{i}(\omega)\left\langle\pi_{K}^{*}, \sigma_{i} \otimes \tau_{\nu}\right\rangle
$$

In fact each irreducible constituent $\sigma_{i}$ of the spin representation $\sigma$ is known to be of the form $\tau_{s \rho-\rho_{k}}$, where $s \in W^{1}=\left\{w \in W\left(\mathfrak{g}_{\mathbf{C}}, \mathfrak{h}_{\mathbf{C}}\right) ; w \Psi \supset \Psi_{k}\right\}$. We can thus rewrite the last equality as

$$
\operatorname{Tr} \theta_{\pi, \nu}(\omega)=\sum_{s \in W^{1}} c_{s}(\omega)\left\langle\pi_{k}^{*}, \tau_{s \rho-\rho_{k}} \otimes \tau_{\nu}\right\rangle
$$

where now

$$
c_{s}(\omega)=\sum_{i \in I_{s}} c^{i}(\omega), \quad \text { with } I_{s}=\left\{i ; \sigma_{i}=\tau_{s \rho-\rho_{k}}\right\} .
$$

Using (2.5) and also the fact that $N_{\Gamma}\left(\pi^{*}\right)=N_{\Gamma}(\pi), \chi_{\pi^{*}}(\Omega)=\chi_{\pi}(\Omega)$, the left-hand side of (2.4) becomes

$$
\lim _{t \rightarrow 0^{+}} t^{m / 2-l} \sum_{s \in W^{1}} c_{s}(\omega) \sum_{\pi \in \hat{G}_{\Gamma, \nu}} N_{\Gamma}(\pi)\left\langle\pi_{K}, \tau_{s \rho-\rho_{k}} \otimes \tau_{\nu}\right\rangle e^{\chi_{\pi}(\Omega) t} .
$$

Thus, the information given by the theorem for the coefficients of the negative powers of $t$ in the asymptotic expansion (0.2) amounts to the following family of equations.

Corollary. For each $\omega \in\left(\bigwedge^{2 l} \mathfrak{p}_{\mathbf{C}}^{*}\right)^{K}$, one has

$$
\sum_{s \in W^{1}} c_{s}(\omega) a_{j}\left(\tau_{s \rho-\rho_{k}} \otimes \tau_{\nu}\right)=0, \quad \text { if } 0 \leq j \leq l-1,
$$

and

$$
\sum_{s \in W^{1}} c_{s}(\omega) a_{l}\left(\tau_{s \rho-\rho_{k}} \otimes \tau_{\nu}\right)=(-1)^{l} C_{m}\left\langle\operatorname{ch}\left(r_{\nu}\right) \wedge \hat{A}(r), \bar{\omega}\right\rangle \operatorname{vol}(\Gamma \backslash G / K)
$$




\section{REFERENCES}

1. D. Barbasch and H. Moscovici, $L^{2}$-index and the Selberg trace formula, J. Funct. Anal. 53 (1983), 151-201.

2. E. Getzler, Psuedodifferential operators on supermanifolds and the Atiyah-Singer index theorem, Comm. Math. Phys. 92 (1983), 163-178.

3. _ A short proof of the local Atiyah-Singer index theorem, Topology 25 (1986), 111-117.

4. R. J. Miatello, The Minakshisundaram-Plejel coefficients for the vector valued heat kernel on compact locally symmetric spaces of negative curvature, Trans. Amer. Math. Soc. 260 (1980), 1-33.

5. __ Alternating sum formulas for multiplicities in $L^{2}(\Gamma \backslash G)$. II, Math. Z. $182(1983), 35-43$.

6. R. Parthasarathy, Dirac operator and the discrete series, Ann. of Math. (2) 96 (1972), 1-30.

7. N. Wallach, An asymptotic formula of Gelfand and Gangolli for the spectrum of $\Gamma \backslash G$, J. Differential Geom. 11 (1976), 91-101.

Department of Mathematics, Ohio State University, Columbus, Ohio 43210 